\title{
ACOMETIMENTO METASTÁTICO LINFONODAL NO CÂNCER COLORRETAL
}

\author{
Túlio PEREIRA Jr., Rejane Aparecida Bueno TORRES e Ana Margarida M. F. NOGUEIRA
}

\begin{abstract}
RESUMO - Racional - No Brasil, o carcinoma colorretal é a terceira causa de óbito por tumores malignos em mulheres e a quinta em homens. O acometimento de linfonodos é um dos principais indicadores de prognóstico destes tumores. Objetivos - Estudar o acometimento linfonodal no carcinoma colorretal de acordo com o comportamento biológico e a topografia do tumor. Material e método - Estudaram-se 185 casos de carcinoma colorretal, analisando-se o acometimento de linfonodos de acordo com topografia, dimensões, angioinvasão e estádio TNM do tumor. Resultados - Dissecaram-se 3906 linfonodos em 185 pacientes (média: 21,1 linfonodo/paciente). Detectaram-se metástases em 399 dos 2573 linfonodos peritumorais (15,5\%) e em 72 dos 1333 linfonodos não-peritumorais (5,4\%). Em 86 pacientes com metástases dissecaram-se 1942 linfonodos, dos quais 471 estavam acometidos (5,45 linfonodo/paciente). Em 26 pacientes observaram-se metástases nos linfonodos peritumorais e não-peritumorais, em 57 somente os linfonodos peritumorais estavam acometidos; em três apenas os linfonodos não-peritumorais eram metastáticos. O número de linfonodos dissecados variou entre os segmentos colônicos acometidos: foi maior no ceco e menor no reto e retossigmóide. O número de linfonodos acometidos correlacionou-se positivamente com profundidade e diâmetro tumoral, acometimento de vasos linfáticos e sangüíneos e negativamente com a resposta linfocítica peritumoral. O estádio linfonodal associou-se significativamente com a profundidade do tumor. Conclusĩes - O carcinoma colorretal acomete principalmente linfonodos peritumorais, mas 29 pacientes $(15,7 \%)$ tinham envolvimento de linfonodos não-peritumorais, o que é importante no estádio e prognóstico do carcinoma colorretal. O estádio linfonodal e o número de linfonodos acometidos relacionaram-se com outros indicadores de prognóstico.
\end{abstract}

DESCRITORES - Neoplasias colorretais. Carcinoma. Linfonodos, patologia. Metástase linfática.

\section{INTRODUÇÃO}

No Brasil, o carcinoma colorretal (CCR) é a terceira causa de óbito por tumores malignos em mulheres e a quinta em homens ${ }^{(18)}$. O acometimento metastático dos linfonodos $(\mathrm{LN})$ regionais é, isoladamente, o fator prognóstico mais importante do $\mathrm{CCR}^{(21)}$, sendo incorporado a todos os sistemas de estádio ${ }^{(2,10,13,29)}$. O acometimento linfonodal metastático relaciona-se a fatores de pior prognóstico, como doença mais avançada e presença de metástase em órgãos à distância ${ }^{(2,4,9,13)}$. Assim, a avaliação de metástases é fundamental no estádio do CCR, sendo de importância primária na decisão de administração de quimioterápicos para o tratamento dos pacientes ${ }^{(17,26)}$.

Nas duas últimas décadas, foram desenvolvidas novas técnicas visando aumento da sensibilidade na detecção de metástase nos LN do CCR. Dentre elas, destacam-se: uso de imunoistoquímica para surpreender micrometástases ${ }^{(12,23)}$, seç̧ões dos LN em vários níveis e soluções para clareamento de gordura, que permitem a dissecção de maior número de $\mathrm{LN}^{(10,23)}$.

Trabalhos recentes com análise de grande número de $\mathrm{LN}$ e, conseqüentemente, aumento da acurácia do exame, demonstraram que o número de $\mathrm{LN}$ livres de acometimento metastático está relacionado com maior sobrevida ${ }^{(10,19,30)}$. A avaliação do perfil qualitativo dos LN tem assumido importância crescente. Nesse contexto, o estudo do LN sentinela $^{(6,10)}(\mathrm{LN}$ que recebe drenagem linfática diretamente da lesão primária), do tamanho dos $\mathrm{LN}^{(10)} \mathrm{e}$ das metástases nos $\mathrm{LN}^{(26,32)}$, da localização dos $\mathrm{LN}$ em relação à topografia da lesão ${ }^{(8)}$, à proximidade do tumor ${ }^{(11)} \mathrm{e}$ de tronco vascular específico ${ }^{(16)}$ têm sido avaliados em relação ao prognóstico. O uso da imunoistoquímica mostrou-se método eficiente na detecção de micrometástases (metástases menores que $2 \mathrm{~mm})^{(6)}$, com impacto no estádio e no prognóstico dos pacientes ${ }^{(6,7,12)}$.

Departamento de Anatomia Patológica e Medicina Legal, Faculdade de Medicina da Universidade Federal de Minas Gerais, Belo Horizonte, MG.

Endereço para correspondência: Dra. Ana Margarida M. F. Nogueira - Departamento de Anatomia Patológica e Medicina Legal, Faculdade de Medicina, UFMG. - Av. Alfredo Balena, $190-5^{\circ}$ andar - 30130-100 - Belo Horizonte, MG. E-mail: anog@medicina.ufmg.br 
Os objetivos deste trabalho foram: a) avaliar o número de LN dissecados e acometidos numa série de CCR; b) correlacionar o acometimento linfonodal com indicadores de comportamento biológico do tumor; c) analisar o acometimento linfonodal em relação à proximidade do tumor.

\section{MATERIAL E MÉTODO}

Estudaram-se CCR de pacientes operados no Hospital das Clínicas da Universidade Federal de Minas Gerais (UFMG), Belo Horizonte, MG, no período compreendido entre 2000 e 2004. As peças cirúrgicas foram analisadas no Serviço de Anatomia Patológica do Departamento de Anatomia Patológica e Medicina Legal da Faculdade de Medicina da UFMG, de acordo com protocolo padronizado em $1998^{(27)}$

O estudo foi aprovado pelo Comitê de Ética em Pesquisa da UFMG (Processo n ${ }^{\circ}$ 327/03).

Os LN regionais foram divididos, de acordo com a sua localização, em dois grupos: peritumorais, definidos como aqueles LN localizados até $5,0 \mathrm{~cm}$ da borda tumoral e os demais foram definidos como não-peritumorais.

Os dados foram levantados a partir das requisições dos exames e dos laudos anatomopatológicos. Excluíram-se do trabalho os casos com tumor de sítio primário desconhecido, os que sofreram remissão após terapêutica neoadjuvante, aqueles com tumores múltiplos ou sem a discriminação da localização (peritumorais e não-peritumorais) dos LN.

O número de LN dissecados e acometidos foi analisado em relação à topografia da lesão, à invasão vascular e linfática e ao estádio $\mathrm{TNM}^{(29)}$. Nas peças de colectomia total foram incluídos os LN relacionados com o segmento do cólon acometido pelo tumor. Dados referentes aos casos analisados entre 2000 e 2002 foram revistos de acordo com a atualização do sistema TNM ${ }^{(29)}$. O CCR foi topografado, de acordo com o sistema da American Joint Committee on Cancer $^{(1)}$, em ceco, cólon ascendente, flexura hepática, cólon transverso, flexura esplênica, cólon descendente, cólon sigmóide, retossigmóide e reto.

A comparação intragrupos foi feita pelos testes nãoparamétricos de Mann-Whitney e Kruskal-Wallis. A comparação entre o número de linfonodos acometidos e diâmetro tumoral e os grupos de estádio foi feita através dos coeficientes de correlação de Pearson e Spearman, respectivamente.

\section{RESULTADOS}

A amostra era composta por 185 pacientes com CCR, em que $81(43,8 \%)$ eram do sexo masculino e $104(56,2 \%)$ do feminino. A idade média dos pacientes foi de $63,1 \pm 14,6$ anos. Predominaram os adenocarcinomas moderadamente diferenciados ( $\mathrm{n}=143 ; 77,7 \%)$.

Em 185 pacientes estudados, dissecaram-se 3906 LN (média: 21,1 LN dissecados/paciente), dos quais $2573 \mathrm{LN}$ eram peritumorais (65,9\%; média: 13,9 LN/paciente) e $1333 \mathrm{LN}$ nãoperitumorais $(34,1 \%$; média: $7,2 \mathrm{LN} /$ paciente). Detectaram-se metástases em 399 dos 2573 LN peritumorais (15,5\%) e em 72 dos 1333 LN não-peritumorais (5,4\%).
Em 99 pacientes $(53,5 \%)$ não se observou acometimento linfonodal. Em 86 pacientes $(46,5 \%)$ com metástases foram dissecados $1942 \mathrm{LN}$, dos quais 471 estavam acometidos (média: 5,45 LN acometidos/paciente). Destes pacientes, em 26 constataram-se metástases nos LN peritumorais e não peritumorais; em 57 somente os LN peritumorais estavam acometidos; em três casos apenas os LN não-peritumorais eram metastáticos.

Houve correlação positiva entre o número de LN acometidos nos grupos peritumoral e não-peritumoral $(P<0,001)$ (Tabela 1). Os três casos com metástase exclusivamente no grupo nãoperitumoral não apresentavam características particulares, exceto pelo pequeno número de $\mathrm{LN}$ dissecados em dois deles (Tabela 2).

TABELA 1 - Correlação entre o número de linfonodos acometidos nos grupos de linfonodos peritumorais e não-peritumorais

\begin{tabular}{lcccccc}
\hline \multirow{2}{*}{$\begin{array}{l}\text { Número de linfonodos } \\
\text { peritumorais positivos }\end{array}$} & \multicolumn{5}{c}{$\begin{array}{c}\text { Número de linfonodos } \\
\text { não-peritumorais positivos }\end{array}$} & \multirow{2}{*}{ Total } \\
\cline { 2 - 5 } & 0 & 1 & 2 & 3 & $>3$ & \\
\hline 0 & 99 & 2 & 0 & 0 & 1 & 102 \\
1 & 22 & 1 & 1 & 1 & 1 & 26 \\
2 & 11 & 2 & 1 & 0 & 1 & 15 \\
3 & 5 & 3 & 0 & 1 & 0 & 9 \\
$>3$ & 19 & 5 & 4 & 0 & 5 & 33 \\
Total & 156 & 13 & 6 & 2 & 8 & 185 \\
\hline$(P<0,001)$ & & & & &
\end{tabular}

TABELA 2 - Características dos casos que apresentaram metástase apenas em linfonodos não-peritumorais

\begin{tabular}{lccc}
\hline Característica & Caso 1 & Caso 2 & Caso 3 \\
\hline Idade do paciente & 44 & 77 & 81 \\
Comprimento do segmento & $12 \mathrm{~cm}$ & $34 \mathrm{~cm}$ & $26 \mathrm{~cm}$ \\
Diâmetro tumoral & $5 \mathrm{~cm}$ & $12 \mathrm{~cm}$ & $3 \mathrm{~cm}$ \\
Grau de diferenciação tumoral & Moderado & Pouco & Moderado \\
Infiltração em vasos linfáticos & Presente & Presente & Presente \\
Infiltração em vasos sangüíneos & Ausente & Presente & Ausente \\
PT & pT3 & pT3 & pT3 \\
Total de linfonodos dissecados & 40 & 11 & 8 \\
Número de linfonodos não-peritumorais com metástase & 4 & 1 & 1 \\
\hline
\end{tabular}

O total de LN dissecados variou nos diferentes segmentos colônicos acometidos (Tabela 3 ). O número de LN peritumorais dissecados em casos de tumores do ceco foi significativamente maior (em média 7,9 LN a mais; $P=0,017$ ) do que aquele observado em tumores do reto e retossigmóide. O total de LN dissecados foi maior nos tumores do cólon ascendente $(n=16$; média $=27,8)$ e menor naqueles da flexura esplênica $(n=06$; média $=14,7)$, mas sem diferença significativa, provavelmente devido ao pequeno número de pacientes com $\operatorname{CCR}(\mathrm{n}=22)$ nestas localizações. Não houve diferença significativa entre os demais grupos de LN e os segmentos do cólon (Tabela 3). 
TABELA 3 - Número de linfonodos dissecados e seu acometimento em relação à localização do tumor nos diversos segmentos colônicos

\begin{tabular}{|c|c|c|c|c|}
\hline \multirow{2}{*}{ Localização do tumor } & \multicolumn{2}{|c|}{ Número de linfonodos peritumorais* } & \multicolumn{2}{|c|}{ Número de linfonodos não-peritumorais* } \\
\hline & Dissecados & Acometidos & Dissecados & Acometidos \\
\hline Ceco $(n=28)$ & $520(18,6 \pm 7,8)$ & $45(1,6 \pm 2,7)$ & $165(5,9 \pm 4,2)$ & $1(0,006 \pm 1,2)$ \\
\hline C. ascendente $(n=16)$ & $302(18,9 \pm 11,3)$ & $15(0,9 \pm 2,1)$ & $142(8,9 \pm 7,7)$ & $1(0,007 \pm 0,3)$ \\
\hline Flex. hepática $(n=6)$ & $68(11,3 \pm 8,2)$ & $28(4,7 \pm 7,9)$ & $48(8,0 \pm 5,9)$ & $3(0,5 \pm 0,8)$ \\
\hline C. transverso $(\mathrm{n}=8)$ & $92(11,5 \pm 6,1)$ & $33(4,1 \pm 8,2)$ & $39(4,9 \pm 5,7)$ & $5(0,6 \pm 0,4)$ \\
\hline Flex. Esplênica $(n=6)$ & $64(10,7 \pm 7,8)$ & $5(0,8 \pm 1)$ & $24(4,0 \pm 6,0)$ & 0 \\
\hline C. descendente $(n=11)$ & $144(13,1 \pm 7,5)$ & $24(2,2 \pm 3,7)$ & $123(11,2 \pm 9,5)$ & $4(0,4 \pm 0,7)$ \\
\hline C. sigmóide $(\mathrm{n}=28)$ & $437(15,5 \pm 8,7)$ & $103(3,7 \pm 5,9)$ & $143(5,1 \pm 6,2)$ & $8(0,3 \pm 0,9)$ \\
\hline Retossigmóide $(\mathrm{n}=29)$ & $386(13,3 \pm 12,9)$ & $64(2,2 \pm 3,2)$ & $239(8,2 \pm 6,4)$ & $9(0,3 \pm 0,9)$ \\
\hline Reto $(n=52)$ & $553(10,6 \pm 9,5)$ & $82(1,6 \pm 3,9)$ & $410(7,9 \pm 7,0)$ & $41(0,8 \pm 2)$ \\
\hline
\end{tabular}

* número de linfonodos (média \pm desvio-padrão)

C. $=$ cólon
Flex = flexura

A média de $\mathrm{LN}$ peritumorais dissecados foi inferior a $12 \mathrm{LN}$ em vários segmentos (Tabela 3). Caso apenas os LN peritumorais fossem utilizados para o estádio, $28(15,1 \%)$ pacientes teriam número insuficiente para um estádio adequado ( $\mathrm{pNx}$ ) no sistema $\mathrm{TNM}^{(29)}$. Com a inclusão dos LN não-peritumorais, apenas $10(5,4 \%)$ casos tiveram número insuficiente de LN para estádio adequado no sistema TNM, sendo classificados como $\mathrm{pNx}$.

O número de LN, dissecados e acometidos, correlacionouse positivamente com diâmetro tumoral $(P \leq 0,03)$. No entanto, quando se analisou isoladamente o subgrupo de $\mathrm{LN}$ peritumorais acometidos, não se observou correlação com o diâmetro tumoral $(P=0,45)$.

O estádio linfonodal $(\mathrm{pN})$ associou-se significativamente com a profundidade do tumor (pT) $(P<0,002)$, conforme evidenciado na Tabela 4 . A análise mostrou que 44 dos 54 pacientes $(81,48 \%)$ com tumores mais superficiais (pT1 ou pT2) eram pN0. Pelo contrário, dos 131 pacientes com tumores mais profundos (pT3 ou pT4), apenas $54(41,98 \%)$ eram pN0.

TABELA 4 - Correlação entre a profundidade da lesão primária (pT)* e o estádio dos linfonodos (pN)

\begin{tabular}{ccccccc}
\hline \multirow{2}{*}{$\mathrm{pT}$} & \multicolumn{5}{c}{$\mathrm{pN}$} & \multirow{2}{*}{ Total de casos } \\
\cline { 2 - 5 } & $\mathrm{pNx}$ & $\mathrm{pN} 0$ & $\mathrm{pN} 1$ & $\mathrm{pN} 2$ & $\mathrm{pN} 3$ & 6 \\
\hline $\mathrm{pT} 1$ & $1 / 1,0$ & $4 / 9,5$ & - & $1 / 16,0$ & - & 48 \\
pT2 & $2 / 4,5$ & $40 / 17,2$ & $4 / 17,5$ & $2 / 22,5$ & - & 117 \\
pT3 & $5 / 7,4$ & $51 / 22,7$ & $27 / 24,7$ & $33 / 25,2$ & $1 / 31,0$ & 14 \\
pT4 & $2 / 10$ & $4 / 20,5$ & $3 / 41,3$ & $5 / 15,6$ & - & 185 \\
Total & $10 / 6,7$ & $99 / 19,8$ & $34 / 25,7$ & $41 / 23,7$ & $1 / 31,1$ &
\end{tabular}

A presença de LN acometidos associou-se negativamente com a resposta linfocítica peritumoral $(P=0,04)$. O número de LN acometidos, totais e peritumorais, relacionou-se significativamente com o acometimento de vasos linfáticos $(P<0,001)$ e sangüíneos $(P<0,001)$. A resposta linfocítica peritumoral apresentou correlação negativa com o número total de $\mathrm{LN}$ acometidos $(P=0,03)$.

\section{DISCUSSÃO}

Há evidências de que o prognóstico e sobrevida de pacientes com CCR dependem principalmente de estádio adequado, especialmente da avaliação de metástases linfonodais ${ }^{(19,30)}$.

O estádio dos LN no CCR foi iniciado por DUKES ${ }^{(13)}$, em 1932, modificado para o sistema ASTLER e COLLER ${ }^{(2)}$ em 1954. Atualmente, tem-se como alternativa o sistema $\mathrm{TNM}^{(29)}$, revisto em 2002 e que utiliza o número de LN envolvidos para estádio.

Tema muito abordado recentemente tem sido o número necessário de LN para estádio acurado dos tumores ${ }^{(17,30)}$. Estudos sugerem um ponto de corte situado entre 6 e $17 \mathrm{LN}^{(10)}$. De acordo com o TNM, em casos sem metástases, são necessários 12 LN para o estádio ${ }^{(10,30)}$. BILCHIK ${ }^{(5)}$ defende que não há grande benefício em se estabelecer um ponto de corte e que, quanto maior o número de $\mathrm{LN}$, maior será a acurácia do estádio ${ }^{(10)}$. Atualmente, sugere-se o uso de substâncias para clareamento de gordura para melhorar a identificação de LN e, assim, aumentar o número de $\mathrm{LN}$ dissecados ${ }^{(28)}$ em situações nas quais o número mínimo de LN para estádio não é atingido ${ }^{(10)}$, conduta que é adotada no Serviço de Anatomia Patológica da Faculdade de Medicina da UFMG.

$\mathrm{O}$ número de $\mathrm{LN}$ estudados sofre interferência de fatores interdependentes, tais como: localização e características histopatológicas do tumor, aspectos inerentes ao paciente, propósito do procedimento (curativo ou paliativo), número de lesões, anatomia vascular de cada segmento $^{(8,20)}$ e do tratamento dispensado aos LN tanto cirúrgico, quanto anatomopatológico ${ }^{(4,10,17)}$. Na presente casuística, o número de LN dissecados (acometidos ou não) foi maior do que em outros estudos $^{(4,19,30,32)}$, demonstrando a qualidade da ressecção cirúrgica e dissecção no Serviço. Na região peritumoral foram identificados $2 / 3$ dos LN dissecados e 79,45\% dos LN acometidos, resultado semelhante ao encontrado na literatura ${ }^{(11)}$. Entretanto, em 29 pacientes $(15,7 \%)$ havia acometimento de LN não-peritumorais, o que é importante, considerando que se observou correlação entre o número total de $\mathrm{LN}$ acometidos e outros indicadores de prognóstico para $\mathrm{CCR}$. O número de $\mathrm{LN}$ não-peritumorais variou com a localização segmentar e com o diâmetro tumoral, possivelmente devido a maior quantidade de material atribuído à 
fração restante ou por características inerentes a cada segmento colônico $^{(8)} \mathrm{e}$ à extensão da cirurgia. $\mathrm{O}$ comprometimento exclusivo de LN não-peritumorais é raro. $\mathrm{Na}$ análise do presente estudo, apenas em três casos observou-se metástase exclusivamente nos LN não-peritumorais, resultado semelhante ao encontrado por CSERNI et al. ${ }^{(11)}$ que, em 100 casos, identificaram apenas 1 paciente com acometimento de LN em frações periféricas e não em proximais. Entretanto, poucos trabalhos analisam o acometimento linfonodal, relacionando a distância dos $\mathrm{LN}$ ao tumor.

Houve associação dos critérios de agressividade tumoral entre si (resultados não apresentados) e com o estádio, o que se relaciona a pior prognóstico. A invasão de vasos sangüíneos, linfáticos e a resposta linfocítica peritumoral, que implicam em prognósticos diferentes, são comumente relacionadas às características específicas do tumor, como seu tamanho e acometimento da parede colônica ${ }^{(14)}$, expressão de mucina ${ }^{(3)}$, diferenciação tumoral ${ }^{(15)}$, tipo histológico ${ }^{(25)}$ e instabilidade de microssatélites $^{(24)}$. Estes pacientes poderiam se beneficiar de ressecções cirúrgicas mais amplas ${ }^{(31)}$.

$\mathrm{O}$ estudo mostrou que o grupo de LN mais impactante no estádio $(\mathrm{pN})$ é o de LN peritumorais, resultado justificado pelo maior número de LN no grupo, pela característica da drenagem linfática ${ }^{(22)}$ (mais próxima do tumor e intensa) e pela provável presença do LN sentinela entre os LN peritumorais ${ }^{(6)}$, sendo a drenagem linfática aberrante incomum no $\operatorname{CCR}(4,0 \%)^{(10)}$. Estudos objetivando a otimização do $\mathrm{pN}$ mostraram que a análise detalhada do LN sentinela implica em estádio mais acurado. Para isso, é utilizado estudo do perfil imunoistoquímico ${ }^{(6)}$, múltiplos cortes (a cada 2 ou $3 \mathrm{~mm}$ ), resultando na detecção de maior número de micrometástases (metástases com até $2 \mathrm{~mm}$ ) e de células metastáticas isoladas ${ }^{(6,26)}$

\section{CONCLUSÕES}

Os CCR acometem, principalmente, $\mathrm{LN}$ peritumorais, mas em $15,7 \%$ dos casos havia acometimento LN não-peritumorais, o que é importante no estádio e prognóstico do CCR. O estádio $\mathrm{pN}$ e o número de $\mathrm{LN}$ acometidos relacionaram-se com outros indicadores de prognóstico.

O estudo mais detalhado dos LN no CCR tem como objetivo melhorar a acurácia do estádio, ao permitir melhor entendimento da biologia das metástases linfonodais.

Pereira Jr T, Torres RAB, Nogueira AMMF. Lymph node evaluation in colorectal cancer. Arq Gastroenterol. 2006;43(2):89-93.

ABSTRACT - Background - In Brazil, colorectal carcinoma is the third cause of death by malignant tumors among women, and the fifth among men. Lymph node involvement is one of the most relevant prognostic maker in these tumors. Aim - To study lymph node involvement in colorectal carcinoma in relationship to biological behavior and tumor location. Patients and Methods - One hundred and eight five colorectal carcinoma cases were studied. Lymph node involvement was analyzed according to tumor location, diameter, vessel invasion, and TNM staging. Results - Three thousand nine hundred and six lymph nodes were harvested in 185 patients (21.1 lymph nodes/patient). Metastasis were detected in 399/2,573 peritumoral lymph nodes (15.5\%) and in 72/1,333 non-peritumoral lymph nodes (5.4\%). Eighty-six patients presented metastasis; in these patients 471/1942 lymph nodes were compromised. In 26 patients peritumoral and non-peritumoral lymph nodes were involved; in 57 cases metastasis were detected only in peritumoral lymph nodes and in 3 , only non-peritumoral lymph nodes were involved. The number of lymph node was higher among cecal tumors and smaller in the rectum and sigmoid. There was a positive correlation between the number of metastatic lymph node and $\mathrm{pT}$, tumor diameter and lymphatic and venous invasion; there was a negative correlation between lymph node involvement and lymphocytic response; $\mathrm{pN}$ was significantly associated with $\mathrm{pT}$. Conclusions - Colorectal carcinoma involves preferentially peritumoral lymph node, but in 29 patients $(15,7 \%)$ non-peritumoral lymph nodes were affected, which is important for tumor staging and prognosis. $\mathrm{pN}$ and the number of metastatic lymph nodes were associated with other behaviour markers.

HEADINGS - Colorectal neoplasms. Carcinoma. Lymph nodes, pathology. Lymphatic metastasis. 


\section{REFERÊNCIAS BIBLIOGRÁFICAS}

1. American Joint Committee on Cancer. Colon and rectum. In: Fleming ID, Cooper JS, Henson DE, Hunter RVP, Kennedy BJ, Murphy GP, O’Sullivan B, Sobin LH, Yarbro JW, editors. AJCC cancer staging manual. 5th ed. Philadelphia: Lippincott-Raven; 1997. p.83-90.

2. Astler VB, Coller FA. The classification significance of direct extension of carcinoma of the colon and rectum. Ann Surg. 1954;139:846-51.

3. Baldus SE, Mönig SP, Hanisch F-G, Zirbes TK, Flucke U, Oelert S, Zilkens G, Madejczik B, Thiele J, Schneider PM, Hölscher1 AH, Dienes HP. Comparative evaluation of the prognostic value of MUC1, MUC2, sialyl-Lewisa and sialyl-Lewisx antigens in colorectal adenocarcinoma. Histopathology. 2002;40:440-9.

4. Baxter NN, Virnig DJ, Rothenberger DA, Morris AM, Jessurun J, Virnig BA. Lymph node evaluation in colorectal cancer patients: a population-based study. J Natl Cancer Inst. 2005;97:219-25

5. Bilchik A. More (nodes) + more (analysis) $=$ less (mortality): challenging the therapeutic equation for early-stage colon cancer. Ann Surg Oncol. 2003;10:203-5.

6. Bilchik BAJ, Nora DT, Sobin LH, Turner RR, Trocha S, Krasne D, Morton DL. Effect of lymphatic mapping on the new tumor-node-metastasis classification for colorectal cancer. J Clin Oncol. 2003;21:668-72.

7. Bukholm IRK, Bondi J, Wiik P, Nesland JM, Andersen SN, Bakka A, Bukholm G. Presence of isolated tumour cells in mesenteric lymph nodes predicts poor prognosis in patients with stage II colon cancer. Eur J Surg Oncol. 2003;29:862-6.

8. Canessa CE, Badia F, Fierro S, Fiol V, Hayek G. Anatomic study of the lymph nodes of the mesorectum. Dis Colon Rectum. 2001;44:1333-6.

9. Cserni G. Nodal staging of colorectal carcinomas and sentinel nodes. J Clin Pathol. 2003;56:327-35.

10. Cserni G. The influence of nodal size on the staging of colorectal carcinomas. J Clin Pathol. 2002;55:386-90.

11. Cserni G, Tarján M, Bori R. Distance of lymph nodes from the tumor an important feature in colorectal cancer specimens. Arch Pathol Lab Med. 2001;125:246-9.

12. Cutait R, Alves VAF, Lopes LC, Cutait DE, Borges JLA, Singer J, Silva JH, Goffi FS Restaging of colorectal cancer based on the identification of limph node micrometastases through immunoperoxidase staining of CEA and cytokeratins. Dis Colon Rectum. 1991;34:917-20.

13. Dukes CE. The classification or cancer of the rectum. J Pathol Bacteriol. 1932;35:323-32.

14. Farhoud S, Bromberg SH, Barreto E, Godoy AC. Variáveis clínicas e macroscópicas que influenciam o prognóstico do carcinoma corretal. Arq Gastroenterol. 2002;39:163-72.

15. Guerrero D, Balen E, Martinez-Penuela JM, Garcia-Foncillas J, Larrinaga B, Caballero MC, Herrera J, Lera JM. Association between microsatellite instability and clinico-pathological characteristics in sporadic colon cancer. Med Clin (Barc). 2005;124:441-6.

16. Hida J, Yasutomi M, Maruyama T, Fujimoto K, Nakajima A, Uchida T, Wakano T, Tokoro T, Kubo R, Shindo K. Indication for using high ligation of the inferior mesenteric artery in rectal cancer surgery. Examination of nodal metastases by the clearing method. Dis Colon Rectum. 1998;41:987-91

17. Horzic M, Kopljar M. Minimal number of lymph nodes that need to be examined for adequate staging of colorectal cancer: factors influencing lymph node harvest. Hepatogastroenterology. 2005;52:86-9.
18. Instituto Nacional do Câncer. INCA. São Paulo: estimativas da incidência e mortalidade por câncer no Brasil 2003. [citado 2004 jul 9]. Disponível em: http://www.inca.gov.br/.

19. Joseph NE, Sigurdson ER, Hanlon AL, Hao Wang, Mayer RJ, MacDonald JS, Catalano PJ, Haller DG. Accuracy of determining nodal negativity in colorectal cancer on the basis of the number of nodes retrieved on resection. Ann Surg Oncol. 2003;10:213-8.

20. Lacerda-Filho A, Silva RG, Queiroz FL, Luz MMP. Estado atual da terapêtica cirúrgica do câncer colorretal. In: Castro LP, Savassi-Rocha PR, Rodrigues MAG, Murad AM. Editores. Tópicos em gastroenterologia 12: câncer do aparelho digestivo. Rio de Janeiro: MEDSI; 2002. p.245-78.

21. Lindmark G, Gerdin B. Prognostic predictors in colorectal cancer. Dis Colon Rectum. 1994;37:1219-27.

22. Marzo AL, Lake RA, Lo D, Sherman L, McWilliam A, Nelson D, Robinson BWS, Scott B. Tumor antigens are constitutively presented in the draining lymph nodes. J Immunol. 1999;162:5838-45.

23. Merrie AEH, van Rij AM, Dennett ER, Phillips LV, Yun K, McCall JL. Prognostic significance of occult metastases in colon cancer. Dis Colon Rectum. 2003;46:221-31.

24. Messerini L, Ciantelli M, Baglioni S, Palomba A, Zampi G, Papi L. Prognostic significance of microsatellite instability in sporadic mucinous colorectal cancers. Hum Pathol. 1999;30:629-34.

25. Park YJ, Park KJ, Park JG, Lee KU, Choe KJ, Kim JP. Prognostic factors in 2230 Korean colorectal cancer patients: analysis of consecutively operated cases. World J Surg. 1999;23:721-6.

26. Perez RO, Habr-Gama A, Nishida-Arazawa ST, Rawet V, Coelho-Siqueira SA, Kiss DR, Gama-Rodrigues JJ. Lymph node micrometastasis in stage II distal rectal cancer following neoadjuvant chemoradiation therapy. Int J Colorectal Dis. 2005;20:434-9. [Epub 2005 Mar 10].

27. Salles PGO, Nogueira AMMF. Câncer colorretal: preparo e análise de peças cirúrgicas. In: Castro LP, Editor. Tópicos em gastroenterologia 11: avanços em coloproctologia. Rio de Janeiro: Editora Médica e Científica; 2001. p.167-74.

28. Scott KWM, Grace RH. Detection of lymph node metastases in colorectal carcinoma before and after fat clearance. Br J Surg. 1989;76:1165-7.

29. Sobin $\mathrm{LH}$, Wittekind $\mathrm{Ch}$. Colon and rectum. In: Sobin $\mathrm{LH}$, Wittekind $\mathrm{Ch}$, editors. TNM classification of malignant tumors. 6th.ed. New York: Wiley-Liss; 2002. p.72-6.

30. Swanson RS, Compton CC, Stewart AK, Bland IK. The prognosis of T3N0 colon cancer is dependent on the number of lymph nodes examined. Ann Surg Oncol. 2003; $10: 65-71$

31. Vieira RAC, Lopes A, Almeida PAC, Rossi BM, Nakagawa WT, Ferreira FO, Melo CA. Prognostic factors in locally advanced clon cancer treated by extended resection. Rev Hosp Clín Fac Med S Paulo. 2004;59:361-8.

32. Wong JH, Steinemann S, Tom P, Morita S, Tauchi-Nishi P. Volume of lymphatic metastases does not independently influence prognosis in colorectal cancer. J Clin Oncol. 2002;20:1506-11. 